\title{
Apoio Automatizado à mediação da aprendizagem baseada em experimentos
}

Silvana Rossy de Brito ${ }^{1,2}$, Thaís L.Tavares dos Santos ${ }^{2}$, Aleksandra do Socorro da Silva ${ }^{1,2}$, Kelle Costa ${ }^{1}$, Elói Luiz Favero ${ }^{1}$

${ }^{1}$ Programa de Pós-Graduação em Engenharia Elétrica (PPGEE) -Universidade Federal do Pará (UFPA)

${ }^{2}$ Núcleo de Tecnologias Interativas de Aprendizagem (NUTEIA) - Instituto de Estudos Superiores da Amazônia - UEPA

\{srossy, thaistavares, aleka, kelle, favero\} @ufpa.br

Resumo. A idéia por trás da atividade experimental é permitir aos estudantes atingirem uma maior autonomia intelectual. Para isso, o estudante deve perceber a importância do refletir após o fazer. Esse artigo discute o problema e propõe um sistema, que pode ser incorporado a Ambientes Virtuais de Aprendizagem para gerenciar as atividades apoiadas por esses ambientes. O sistema proposto permite definir processos do tipo "padrão" com atividades que são "instanciadas" para os cursos e conta também conta com uma ferramenta de avaliação automática de textos, que fornece, para o professor, indicadores que podem ser usados para agilizar o feedback para os estudantes.

Palavras-chave: Ambientes Virtuais de Aprendizagem, Aprendizagem Baseada em Experimentos, Mediação da Aprendizagem.

\section{Introdução}

As dificuldades encontradas no processo de aprendizagem quando este não envolve aplicações práticas desafiam pesquisadores e professores. $\mathrm{O}$ investimento em pesquisa e tecnologia para apoiar a aprendizagem experimental transcende os espaços das escolas e já pode ser observado em parques como o TECHNIQUEST (2005) na Inglaterra, que possui a proposta de apresentar aos estudantes um olhar prático para a ciência, oferecendo pequenos simuladores e micromundos que despertam a criança para um tipo de aprendizagem significativa (Ausubel, 1980) e instigante.

Atividades experimentais favorecem a autonomia intelectual (Ferreira e Hartwig, 2004), mas para isso, o enfoque investigativo deve ser adequadamente orientado. Segundo Blümke (2002), metodologias de caráter experimental contribuem para que o professor deixe de impor ao estudante o seu conhecimento e passe a ajudá-lo a crescer como cidadão e a desafiá-lo a buscar o saber científico como uma conquista pessoal. $\mathrm{O}$ estudante, ao deixar de receber os conceitos prontos e acabados, aprende a buscar respostas e encontrar soluções para os problemas propostos com liberdade e responsabilidade, ou seja, desenvolvendo sua autonomia.

Este trabalho está situado no contexto da experimentação como forma de potencializar a aprendizagem. Propõe um sistema que permite definir "processos" tipo "padrão", a exemplo de padrões pedagógicos (Bergin et al., 2005), que podem ser instanciados para cursos apoiados por Ambientes Virtuais de Aprendizagem (AVAs). As atividades dos processos podem ser definidas por professores e estudantes e realizadas por estudantes. No sistema proposto, os professores contam com interface para mediação e acompanhamento do processo, bem como com uma ferramenta para apoio à avaliação automática de textos. 
O artigo está estruturado da seguinte forma: a seção 2 descreve a experimentação didática, os problemas enfrentados e o papel do professor na mediação da aprendizagem; a seção 3 apresenta diferentes formas de conduzir as atividades de experimentação; a seção 4 apresenta um modelo de processo para a experimentação didática com o apoio das tecnologias; a seção 5 apresenta o sistema para apoiar o processo de experimentação didática, com recursos para apóia à mediação; e, finalmente, a seção 6 apresenta as considerações finais.

\section{Aprendizagem baseada em experimentos}

A aprendizagem baseada em experimentos favorece o desenvolvimento de atitudes e destrezas cognitivas de alto nível intelectual em oposição a destrezas manuais ou técnicas instrumentais (Galiazzi et al., 2001). Portanto, realizar uma atividade com experimento não significa apenas executá-la, sem uma ampla discussão de seus resultados, como também não deve ser visto como um simples reforço das aulas teóricas ou para confirmar algo que o estudante já "aprendeu" em sala de aula. Para Blümke (2002), a experimentação, pelo seu potencial, é mais útil se estiver integrada à proposta de ensino e não como mero complemento das atividades.

Assim, o experimento é visto não como uma simples montagem e um conjunto de resultados, mas como um momento de trabalho, reflexão, análise, questionamento, interpretação, troca de idéias, tomada de decisões e de conclusões, mesmo que provisórias (Blümke, 2002). Mais do que uma atividade que provoca a curiosidade, o experimento deve ser considerado uma importante prática pedagógica desenvolvida pelo estudante. Para Blümke (2002), a realização de um experimento deve incluir intercaladamente, tarefas teóricas e experimentais onde o fazer é importante, mas o refletir é fundamental (Blümke, 2002).

Nessa direção, as pesquisas, apoiadas por teorias psicológicas e pedagógicas conduzem a processos que colocam os estudantes no centro do processo de aprendizagem, e que levam em conta as necessidades individuais, os interesses, as capacidades e os estilos, encorajando a aprendizagem significativa (Ausubel 1980), levando em conta os valores inter-pessoais. Segundo Shang et al (2001), a ênfase crescente na aprendizagem construtivista centrada no estudante rendeu uma verdadeira revolução nas teorias e práticas educacionais: os estudantes devem estar intrinsecamente motivados na exploração, de forma que desenvolvam as habilidades de desafiar e atividades de resolver problemas. Como forma de tornar a aprendizagem mais ativa, Shang et al. (2001) descrevem algumas práticas: ampliar as experiências, podendo-se criar pequenos grupos de estudantes, colocando-os em situações de tomadas de decisão ou sugerindo questões; levar vantagem do "poder de interação", de forma que as várias atividades de aprendizagem possam multiplicar o impacto educacional. Por exemplo, se os estudantes escrevem seus próprios pensamentos sobre um tópico, esse texto pode sugerir uma pequena discussão de grupo, que pode ser valiosa para outros. Nesse ponto, a observação é mais rica e atrativa, mesmo para aqueles que não participam do diálogo. Essa multiplicação pode acontecer se, após a construção de algum artefato, os estudantes escrevem sobre a sua experiência e discutem com os outros suas observações. Relacionar a experiência e o diálogo pode dar aos estudantes uma perspectiva nova sobre as suas convicções e valores, ajudando os estudantes a construir os muitos possíveis significados para as suas experiências.

As observações de Shang et al. (2001) sugerem que as estratégias para tornar a aprendizagem significativa envolvem algum tipo de experiência ou diálogo, sendo: dois tipos de diálogo, considerando o diálogo com si mesmo (pensar reflexivamente) e diálogo com outros; dois tipos de experiência, considerando o observar e o fazer.

Cada um dos quatro modos de aprender (pensar, dialogar com outros, observar e 
fazer) tem seu próprio valor, e a sua combinação deles agrega valor ao processo de aprendizagem, tornando-o mais atrativo e significativo para os estudantes.

\subsection{Problemas enfrentados com as atividades experimentais}

No contexto das disciplinas que utilizam a experimentação, tais como a Física, Química, Biologia e Engenharias, problemas como o tempo de aula reduzido fazem com que, após a realização de um experimento, o estudante frequentemente não tenha a oportunidade de analisar os conceitos abordados na experiência feita (Blümke, 2002). É importante encontrar meios para estimular o estudante a observar os fatos ocorridos durante a experiência nas aulas práticas de laboratório favorecendo o momento de análise e síntese da experiência.

No contexto da avaliação das atividades experimentais, existem várias maneiras de como um professor pode avaliar o desempenho de um estudante (Knox et al., 1996): fazer perguntas sobre o experimento dentro ou fora do laboratório; fornecer novas atividades baseadas no experimento; ler um relato de um estudante sobre atividades realizadas, avaliando sua conclusão sobre o experimento; e, avaliação de um colega pelo grupo. A avaliação é importante porque pode estimular os estudantes a refletir sobre os experimentos. Portanto, solicitar que os estudantes mantenham um relato sobre seus trabalhos é uma forma de estimular sua reflexão.

Entretanto, um elevado número de estudantes acompanhados pelo mesmo professor durante a aprendizagem experimental dificulta a mediação da aprendizagem. Nos casos onde são solicitadas "sínteses" escritas pelos estudantes, há uma sobrecarga de trabalho para o professor na avaliação das mesmas e uma conseqüente demora no feedback do professor para essas sínteses.

No sistema proposto, através do registro das experiências, é possível valorizar as atividades de síntese e avaliação; a compreensão através das trocas de informações entre professores e estudantes; o processo de análise através da observação das sínteses realizadas e dos resultados dos experimentos. Os termos síntese, avaliação e análise fazem referência à taxonomia de Bloom et al. (1974).

\subsection{O papel do professor na experimentação didática}

Para apoiar o processo de experimentação didática, deve-se levar em consideração que o professor tem a liberdade de decidir qual a melhor estratégia para conduzir os estudantes durante a aprendizagem. Pode, por exemplo, sugerir uma etapa que favoreça a validação de resultados do experimento (Tavares et al., 2001), que pode ser realizada pelo professor ou pelos estudantes, confrontando-se as sínteses frente a novos dados em uma sessão de autocrítica e de auto-avaliação, na qual todos os experimentos da turma são examinados. O professor pode, por exemplo, sugerir uma ou mais fases de análise do experimento antes de partir para a formalização da atividade realizada. Pode, ainda, adotar modelos cíclicos em que, após a fase final de validação de resultados, o processo volte à fase de identificação do problema, permitindo que o estudante vá amadurecendo seus conceitos a cada iteração do ciclo.

No trabalho experimental, o importante é a reflexão advinda das situações nas quais o material é empregado e, consequentemente, a maneira como o professor integra o trabalho prático na sua formação (Blümke, 2002). Os experimentos não devem se resumir à utilização de instrumentos e deve ser possível confrontar as concepções trazidas pelos estudantes com aquelas desejadas pelos professores.

\section{Diferentes formas de conduzir a aprendizagem através da experimentação}

A idéia de estruturar a aprendizagem experimental de diferentes formas é reforçada pela definição de padrões pedagógicos, que são modelos aplicáveis nos mais 
diferentes contextos da aprendizagem. Especificamente, para o contexto da aprendizagem baseada em experimentos, Bergin et al. (2005) coletaram e revisaram doze modelos existentes na literatura e que se caracterizam por orientar o professor na condução do processo. Dentre esses modelos, estão (Bergin et al., 2005): Ver antes de escutar, que consiste em evitar que os estudantes ouçam coisas como " $<$ isso $>$ é o que irá acontecer quando você fizer <isso>". Esse padrão sugere que se dê aos estudantes a oportunidade de ver e experimentar um novo conceito antes de ouvir sua definição, encorajando a reflexão e minimizando as aulas teóricas; Exponha o Processo, que destaca o processo, mostrando os pontos de decisão críticos, documentando soluções alternativas, mesmo que não conduzam ao resultado desejado; Round Robin, onde cada membro da equipe contribui com uma idéia e o professor deve escrever a idéia no quadro - o objetivo é permitir que o grupo evolua em um tempo definido.

Além dos padrões descritos, Bergin et al. (2005) citam outros, tais como "um conceito, várias implementações", "construir e manter a confiança", "Missão Impossível" e finalmente o padrão "Projeto do Estudante", baseado na abordagem de projetos, reforça a necessidade de uma equipe de trabalho para análises críticas e deve ser mediado para evitar atrasos no cronograma. Esses padrões remetem a uma visão das atividades experimentais como um processo cronologicamente definido e mediado por professores, monitores e especialistas e estruturado com o apoio de ferramentas e artefatos diversos, produzidos ou não pelos estudantes.

\section{Modelos de processo para a experimentação didática}

Diversas propostas sugerem o uso de tecnologias para apoiar o ensino experimental. Como exemplo, sistemas de software como Ambientes Virtuais de Aprendizagem (AVAs) podem permitir que os estudantes comentem experimentos de outros estudantes. Segundo Knox et al. (1996), a Internet permite tornar disponível o material usado nas aulas e os estudantes podem comentar o material disponível. O desafio tem sido decidir onde tais comentários ser localizados, que formato devem possuir e se devem ser editados. Comentários sem importância ou anônimos devem ser evitados, mas críticas construtivas que irão resultar em melhorias devem ser incentivadas.

Nessa proposta, a modelagem do processo de experimentação é baseada em uma analogia entre o processo de cursos e o processo de software. Essa analogia já foi realizada por outros autores (Tavares et al, 2001), (Cesarine, 2004), (Marshall \& Mitchell, 2004) e (Dahmer et al, 2005). As atividades experimentais também são formalizadas em um processo "modelo" proposto por De Jong (citado por Ferreira e Hartwig, 2004).

Como exemplo de uma possível estruturação do processo de experimentação, a figura 1 apresenta o processo com o apoio da tecnologia e envolvendo diferentes papéis: estudantes, como aprendizes, participantes de todo o processo; assistentes de laboratório (monitores), cujos deveres normalmente incluem fornecer ajuda em um nível básico aos estudantes com questões sobre os instrumentos para o experimento; colaboradores ou assistentes já graduados, que podem participar quando um nível moderado de interação é necessário, agindo como uma ligação entre o professor e o estudante e participando como revisores, reforçando conceitos discutidos nas aulas teóricas; especialistas, que podem ser professores ou participantes externos. 


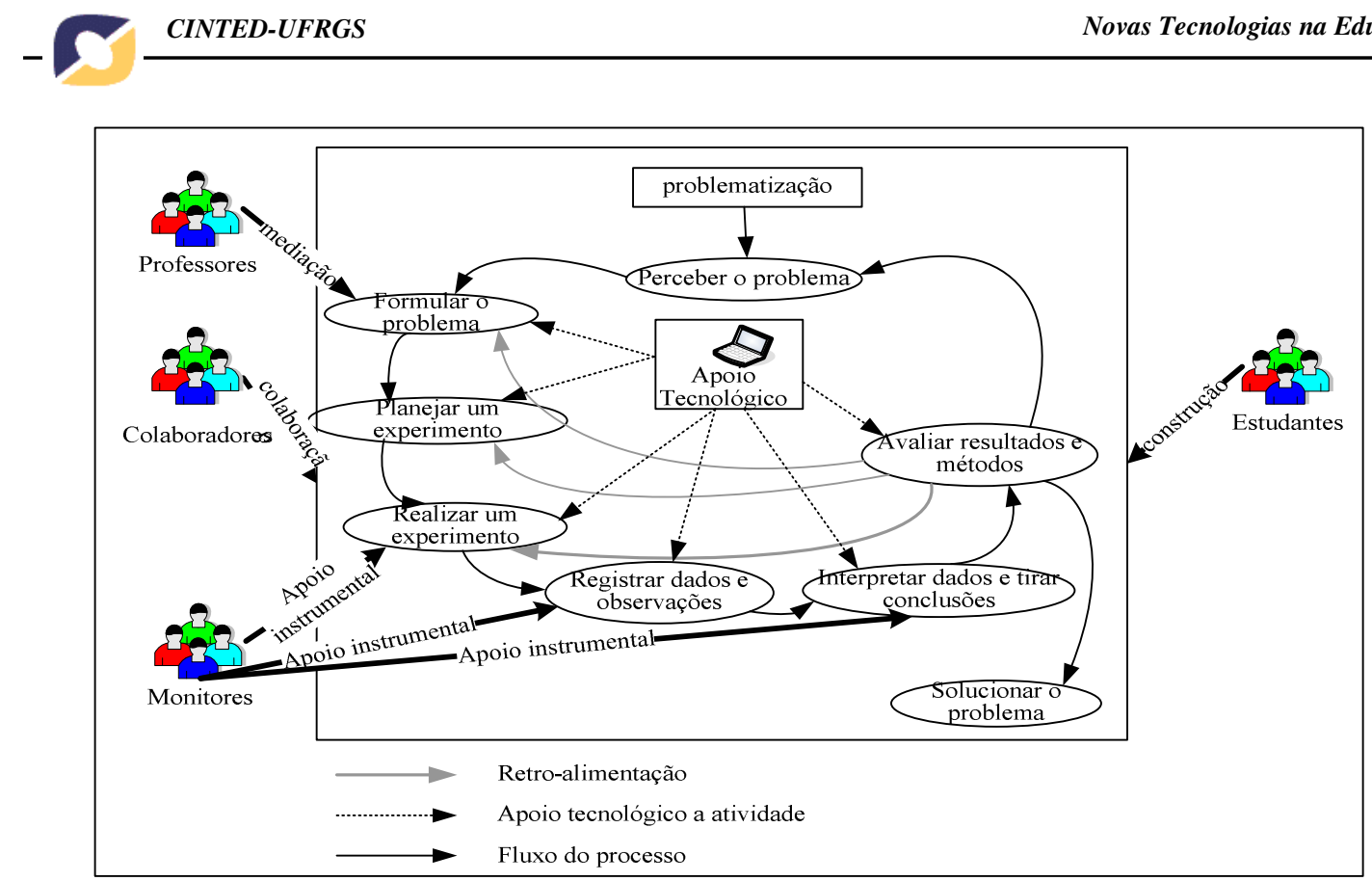

\section{Figura 1. Possível estruturação do processo de experimentação didática com o apoio de tecnologias}

O processo ilustrado na figura 1 inicia com uma fase de problematização onde são levantadas as questões significativas acerca do problema. A atividade inicia onde os estudantes percebem o problema e, em seguida, o formulam (formalizam o problema). O planejamento do experimento segue a formulação do problema e implica na definição dos objetivos uma vez que o experimento deve fornecer os elementos necessários para a resolução do problema. A realização do experimento implica na utilização dos materiais conforme o objetivo definido. Uma vez que o experimento tiver sido realizado, os estudantes devem registrar os dados (de entrada e saída) e as observações que o estudante (ou membro do grupo) julgar necessário. Esse relato é a base para a interpretação dos dados e para que os estudantes tirem conclusões sobre o experimento realizado. Finalmente, a atividade seguinte direciona os estudantes para a avaliação de resultados e métodos, sendo que a avaliação pode envolver a participação de outros estudantes. A etapa de avaliação não implica no término do processo, já que, a partir dela, a percepção do problema pode ser revista pelos estudantes.

Durante todo o processo, enquanto os estudantes participam ativamente do processo (indicado pela relação de construção do estudante com as atividades representadas), o professor atua como mediador em cada uma das etapas. Os colaboradores, tais como especialistas e profissionais podem contribuir com sugestões e, finalmente, os monitores atuam com o apoio instrumental.

\section{Sistema de apoio ao processo de experimentação didática}

Para compreender o funcionamento do sistema, a figura 2 apresenta um metamodelo para processos, que captura os principais conceitos associados às atividades executadas. 


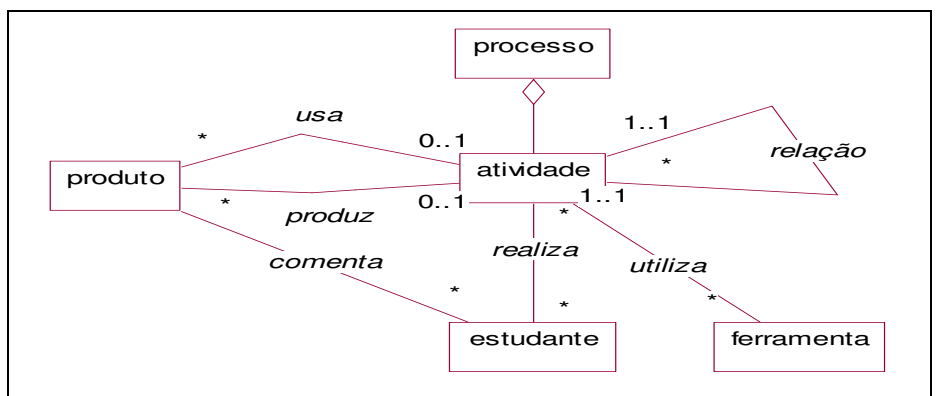

Figura 2. Meta-modelo para o processo de experimentação.

$\mathrm{Na}$ figura 2, adaptada de um modelo para automatização de processos de software (Mafra et al., 2004), cada processo é composto de atividades que podem ter dependência da finalização de outras atividades. As atividades são executadas pelos estudantes, sendo que cada atividade, durante a sua execução, pode consumir ou gerar artefatos (produtos) que, por sua vez, podem ter sido gerados em outras atividades e podem produzir novos produtos. Um produto de uma atividade pode ser uma síntese escrita pelo estudante ou pode ser a descrição do experimento ou dos resultados do experimento. As atividades são apoiadas por ferramentas (de software ou não).

$\mathrm{Na}$ atual versão do sistema, professores e estudantes descrevem as atividades (figura 3) e os alunos executam. Uma atividade torna-se disponível para o estudante logo após ele entregar a documentação de sua atividade predecessora, no caso dessas atividades estarem relacionadas como "término-início". No caso de uma relação do tipo"início-início", a atividades ficam imediatamente disponíveis para o estudante.

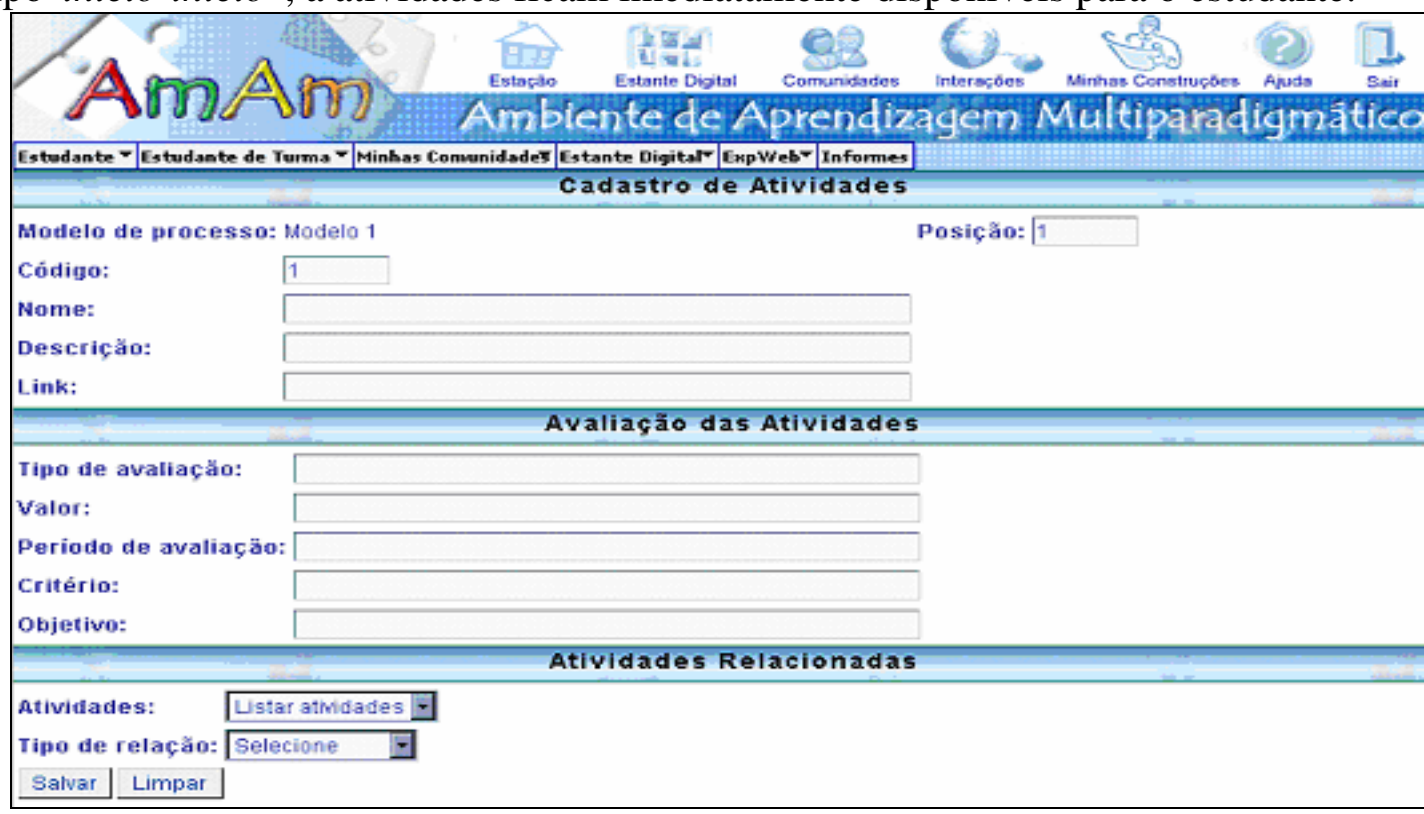

Figura 3. Cadastro de Atividades para um processo “padrão".

Parte da tela de acompanhamento do processo, acessível pelo professor, é apresentada na figura 4. Nessa tela, as quatro atividades cadastradas (Descrição do problema, Planejamento, Realização e Síntese) aparecem para o professor (a lista de atividades é elaborada dinamicamente) juntamente com os seguintes indicadores: execução da atividade (se a atividade foi concluída ou está em construção, ou foi entregue com atraso); feedback (enviado, não enviado, não solicitado); comentário de outros estudantes (comentado ou não comentado); dúvida (enviada ou não enviada). 

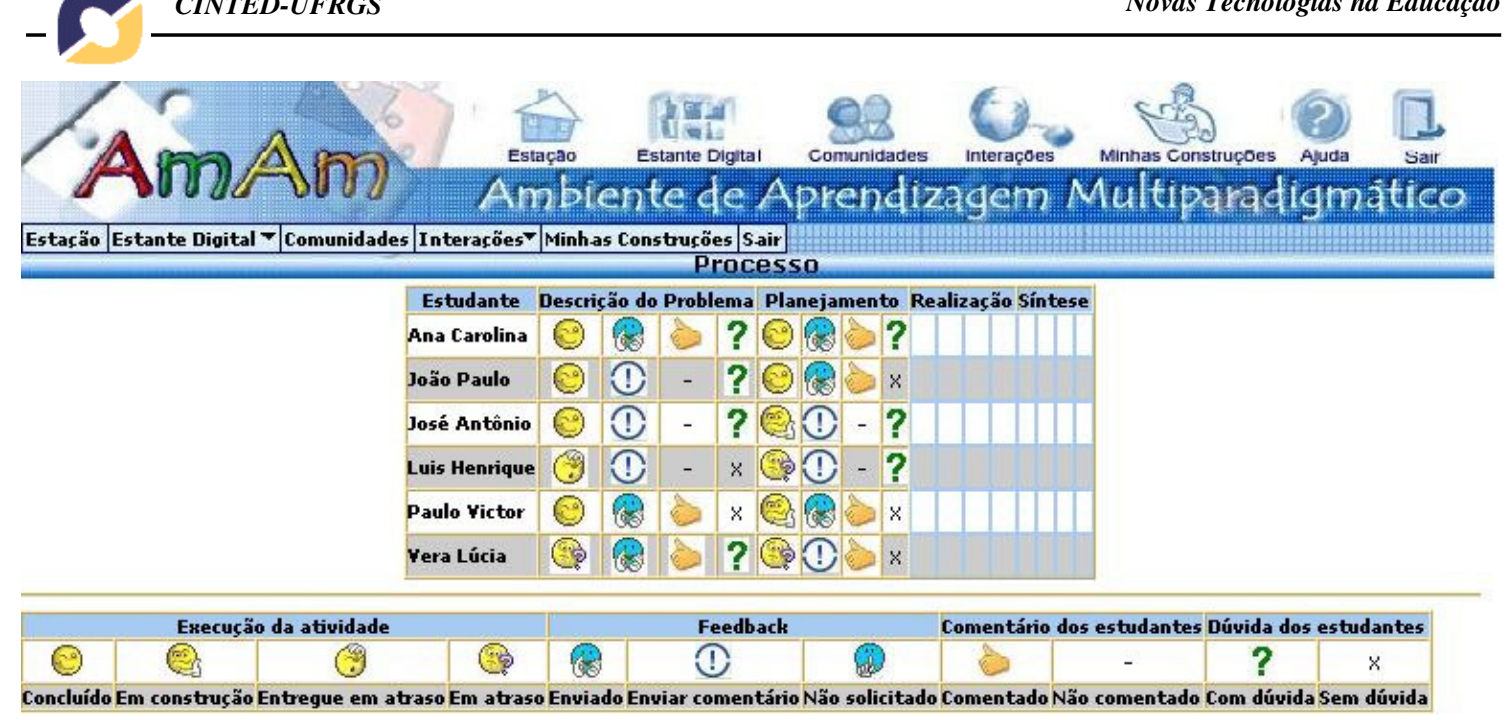

Figura 4. Tela (parcial) de acompanhamento do processo de experimentação.

No processo apresentado, professores e estudantes definiram quatro atividades com prazos limite, durante as quais os estudantes podem preencher um relatório, receber o feedback do professor, fazer perguntas (para o professor ou para a turma) e comentar as atividades de outros estudantes. Na tela do professor, apresentada na figura 3, é possível acessar os relatórios individuais, escrever feedback para os estudantes, visualizar os comentários do grupo para a atividade daquele estudante e visualizar as perguntas individuais que o estudante registrou durante a realização da atividade. Cada atividade tem início e término documentado, de forma que o professor pode acompanhar o estudante e inferir, pelo atraso, quando o mesmo está com problemas na realização da atividade.

Embora na figura 3 apenas 4 atividades sejam apresentadas, o número e a descrição das atividades são definidos pelos professores, em conjunto com os estudantes. Para cada atividade, o estudante tem a liberdade de desenvolver uma descrição (síntese, relato, descrição formal do experimento), realizar perguntas, visualizar o feedback do professor ou comentar as atividades de outros estudantes. No exemplo apresentado, os alunos utilizaram para a experimentação uma ferramenta de software para obtenção dos coeficientes de transmissão e de reflexão de ondas eletromagnéticas em regiões descontínuas (Santos, 2003). No experimento foi utilizado o software MATLAB para a simulação de ondas eletromagnéticas e os valores do campo magnético e do campo elétrico, de acordo com as equações de Maxwell, são calculados automaticamente para a obtenção dos coeficientes de reflexão e transmissão. O objetivo deste experimento é observar a propagação de uma onda eletromagnética incidindo na interface de separação dos meios.

\subsection{Apoio automatizado à mediação}

Para auxiliar na avaliação dos textos produzidos pelos estudantes, o professor conta com o recurso de visualizar a lista dos textos produzidos recuperados pelo sistema. O sistema organiza os documentos de acordo com o modelo de recuperação conhecido como espaço-vetorial (Salton et al., 1975).

Embora a proposta do modelo vetorial tenha surgido em 1968 (Salton, 1969) tendo como objetivo a solução de problemas de busca, é utilizado por ter bom desempenho na recuperação dos dados, visto que leva em consideração o casamento parcial e a proximidade dos documentos em relação aos termos da consulta. Na implementação do modelo, o vocabulário contido nos textos dos estudantes é representado por vetores, cujos elementos são conceitos indexadores, bem como os 
termos contidos na base de descrições de atividades, produtos e ferramentas que também compõe conceitos indexadores. Os vetores são posicionados em um espaço com $t$ dimensões (sendo $t$, o número de termos indexadores) (Salton et al., 1975). A comparação é feita entre os vetores e o grau de relevância é relativo à distância entre os vetores, sendo o cosseno do ângulo entre os vetores a função de comparação usada. Como resultado da aplicação do método, o professor visualiza as sínteses dos estudantes na ordem decrescente da relevância indicada pelo método.

Os textos através dos quais os vetores são montados estão presentes no quadro 1 e o resultado é apresentado na tela de acompanhamento do professor (figura 5).

Quadro 1. Textos analisados pelo método espaço-vetorial.

\begin{tabular}{|l|l|l|l|}
\hline id & Texto & Descrição \\
\hline (a) & $\begin{array}{l}\text { Foi usado o Matlab para propagações } \\
\text { eletromagnéticas o ambiente apresenta o meio 1, } \\
\text { e o meio 2 }\end{array}$ & $\begin{array}{l}\text { Texto produzido por um } \\
\text { estudante após o experimento }\end{array}$ \\
\hline (b) & $\begin{array}{l}\text { Foi usado o Matlab para simulação de ondas } \\
\text { magnéticas o ambiente apresenta o meio 1, que é } \\
\text { um espaço livre e o meio 2 que é um espaço } \\
\text { qualquer }\end{array}$ & $\begin{array}{l}\text { Texto produzido por um } \\
\text { estudante após o experimento }\end{array}$ \\
\hline (c) & $\begin{array}{l}\text { Foi usado o Matlab para propagações } \\
\text { eletromagnéticas o ambiente apresenta o meio 1, } \\
\text { e o meio 2 que é um espaço qualquer }\end{array}$ & $\begin{array}{l}\text { Texto produzido por um } \\
\text { estudante após o experimento }\end{array}$ \\
\hline (d) & $\begin{array}{l}\text { Foi usado Matlab simulação ondas } \\
\text { eletromagnéticas ambiente apresenta meio 1 } \\
\text { espaço livre e meio 2 espaço qualquer }\end{array}$ & $\begin{array}{l}\text { Conteúdo do vetor contendo } \\
\text { as palavras do professor para } \\
\text { descrever o experimento }\end{array}$ \\
\hline
\end{tabular}

Através dessa interface (figura 5) o professor tem acesso aos textos produzidos pelos estudantes, podendo modificar ou incluir um comentário, que pode ser visualizado pelo estudante.

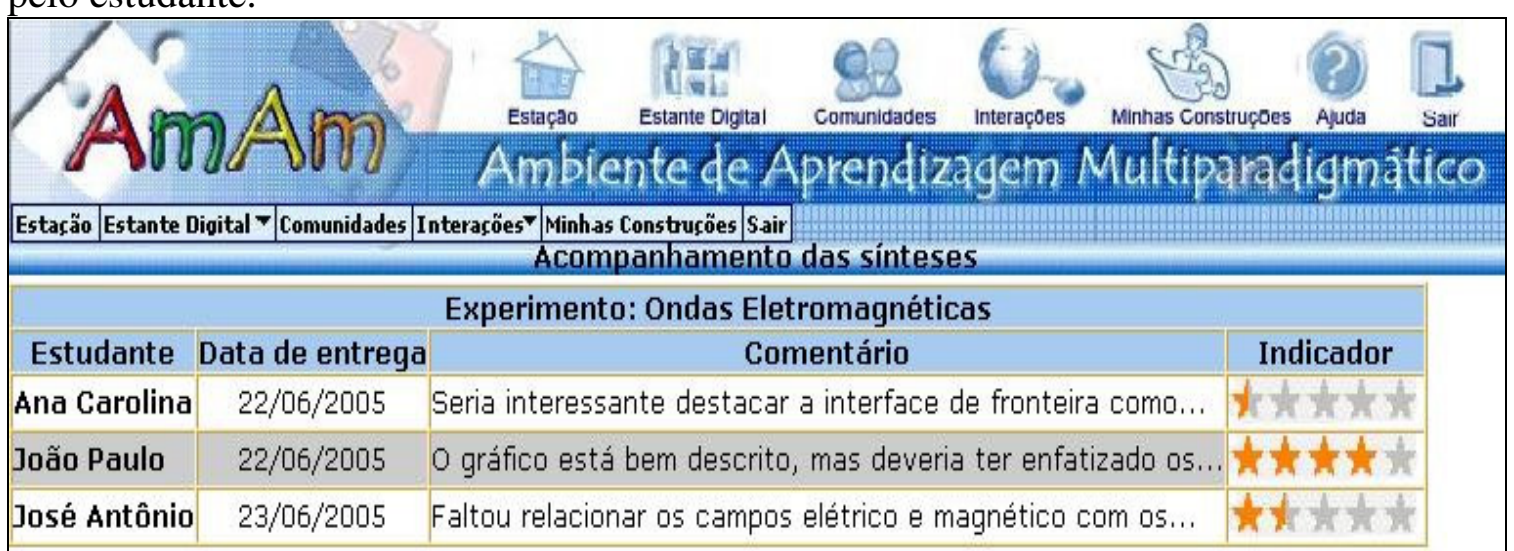

Figura 5. Tela (parcial) do professor para acompanhamento das sínteses.

Embora o método de comparação utilizado apresente dificuldades para lidar com sinônimos e relacionamentos frasais pois não considera a relação entre os termos, os valores resultantes servem para indicar ao professor que termos técnicos essenciais não foram citados nos textos do estudante. Com frequiência, os termos técnicos, que são essenciais, não sofrem alterações, o que aproxima o resultado do esperado. Com a técnica espaço-vetorial é possível acompanhar graficamente a relação de similaridade entre os textos, de forma que seja possível fornecer elementos para que o professor, ainda em laboratório ou, pelo menos, em menor tempo esperado, possa fornecer feedback para as reflexões dos estudantes, representadas nos textos produzidos por eles antes, durante e após a realização do experimento. 
Nos testes realizados, com uma base de dados de até dez sínteses dos estudantes, o modelo espaço-vetorial se mostrou apropriado, apresentando o ranking das sínteses na mesma ordem com que o professor avaliou, embora os textos utilizados para teste tenham sido editados para eliminar erros gramaticais que poderiam comprometer os resultados. Os valores resultantes (variações entre 0-1) da aplicação do método sobre os textos produzidos pelos estudantes são mapeados automaticamente para o indicador do professor em escala de 0-5 estrelas (figura 5).

O sistema está sendo desenvolvido para ser incorporado a ambientes virtuais de aprendizagem (AVA) de forma que estudantes e professores possam utilizá-lo com outras ferramentas disponíveis. É desenvolvido com tecnologia PHP x MySQL para a interface Web, sendo as classes para recuperação de informação (modelo espaço vetorial) implementadas em JAVA. Foram simulados padrões que se encaixam nos modelos "Round Robin" "Projetos do Estudante" e no modelo cujas atividades são apresentadas na figura 1. Nesse momento, o sistema está sendo adaptado para implantação no AmAm (Harb et al., 2003). Para incorporação ao AVA foi criada uma função de "instanciar modelo de processo" que "copia" as atividades do modelo para as atividades dos estudantes, fazendo o ajuste das datas de inicio e fim da atividade.

\section{Conclusões}

No ensino experimental, é importante que o estudante perceba a aprendizagem como um processo de se fazer perguntas muito mais do que encontrar respostas prontas, elaboradas por outros. Para isso, o estudo apresentado aqui busca incentivar a tomada de consciência progressiva por parte dos estudantes e professores acerca da importância do processo, muito mais do que da experimentação em si. A aprendizagem deve estar centrada no estudante, de forma que a perceba como uma tarefa autônoma pela qual deve se tornar responsável.

Sistemas de apoio ao processo conduzem estudantes e professores à reflexão sobre o processo e ao planejamento da aprendizagem como uma tarefa cooperativa. Em oposição ao registro apenas do resultado final, como exemplos e atividades completas, os sistemas com foco em processo tendem a registrar as decisões, os caminhos tomados e as alternativas existentes. Transformar estudantes passivos em ativos implica em tornar viva a reflexão do processo de aprendizagem.

O sistema foi elaborado com base na especificação do processo de experimentação didática com $\mathrm{o}$ apoio de tecnologias. Parte dos problemas foi identificada a partir da literatura especializada em experimentação didática e os requisitos foram capturados com base nesses problemas. Entretanto, na atual fase de desenvolvimento e para efeito de elicitação de novos requisitos para o sistema, um questionário foi elaborado e está sendo aplicado a professores da rede pública e privada, que utilizam abordagem da experimentação no sentido de tornar a ferramenta proposta neste trabalho mais flexível e abrangente, atendendo diferentes métodos (ou padrões) pedagógicos adotados. Para efeito de reduzir o tempo das ações de feedback, outros métodos para recuperação de informação devem ser implementados e testados. Esses recursos tendem a minimizar a sobrecarga do professor na avaliação das construções dos estudantes, além do que, quando alcançado um nível aceitável de precisão, podem ser fornecidos diretamente para o estudante, como ferramenta de apoio à auto-avaliação.

Além das restrições quanto ao método de recuperação de informação utilizado, o modelo e as tecnologias utilizadas ainda apresentam várias limitações, que devem ser minimizadas em trabalhos futuros, como o uso de agentes de software (Russell e Norvig, 1995) para gerenciar e executar o processo, permitindo, por exemplo, que mudanças no processo sejam realizadas em tempo de execução. Deve ser possível que o professor possa definir processos incompletos, refinados ao longo da aprendizagem, 
além de tratar a formação de grupos, com informações mais precisas sobre as características dos estudantes e suas afinidades para atividades cooperativas. Embora os testes tenham sido executados com textos reais produzidos por estudantes, o sistema deve ser efetivamente utilizado e avaliado por um número significativo professores e estudantes.

\section{Referências}

Ausubel, P., Novak, J. D., Hanesian, H. Psicologia Educacional. Rio de Janeiro: Interamericana. 1980.

Bergin, J., Marquardt, K., Manns, M. L., Eckstein, J., Sharp, H., Wallingford, E. Patterns for Experiential Learning. The Pedagogical Patterns Project. http://www.pedagogicalpatterns. org/current/experientiallearning.pdf. Maio, 2005.

Bloom, B. S., et al. Taxonomia dos objetivos educacionais. Porto Alegre: Globo, 1974.

Blümke, R. A. A Experimentação no Ensino de Física. Universidade Regional do Noroeste do Estado do Rio Grande do Sul - Departamento de Física, Estatística e Matemática. Ijuí, 2002.

Cesarini, M., Monga, M., Tedesco, R. Carrying on the eLearning process with a Workflow Management Engine. In ACM Symposium on Applied Computing (SAC'04). Nicosia, Cyprus, 2004..

Dahmer, A., Viccari R.M., Nunes, D.J. A Proposal of Criteria for Evaluation of Distance Education Courses, In The Fourth IASTED International Conference on Web-Based Education. Grindelwald, Switzerland, 2005.

Ferreira, L. H., Hartwig, D. R. Grupo de Trabalho 2 - Experimentação. I Encontro Paulista de Pesquisa em Ensino de Química, Universidade de Campinas - Instituto de Química. http://gpquae.iqm.unicamp.br/EPPEQ.pdf. Fevereiro, 2004.

Galiazzi, M. C., Gonçalves, F. P. A Natureza Pedagógica da Experimentação: Uma Pesquisa na Licenciatura em Química. Química Nova, Vol. 27, No. 2, 326-331, 2004.

Galiazzi, M. C., Rocha, J. M. B., Schmitz, L. C., Souza, M. L., Giesta, S., Gonçalves, F. P. Objetivos das Atividades Experimentais no Ensino Médio: a pesquisa coletiva como modo de formação de professores de ciências. UFRGS (Departamento de Química). 2001.

Harb, M. P. A. A., Brito, S. R., Silva, A. S., Favero, E. L., Tavares, O. L., Francês, C. R. L. AmAm: ambiente de aprendizagem multiparadigmático. In: Simpósio Brasileiro de Informática na Educação, Rio de Janeiro: NCE-IM-UFRJ. p. 223-232, 2003.

Knox, D., Wolz, U., Joyce, D., Koffman, E., Krone, J., Laribi, A., Myers, J. P., Proulx, V. K., Reek, K. A. Use of laboratories in Computer Science education: guidelines for good practice. ACM - Association for Computing Machinery. USA. 1996.

Mafra, S.N., Barros, M., Travassos, G. H. EnactPro: Automatizando processos de software. In: XI Sessão de Ferramentas, Procedings on Simpósio Brasileiro de Engenharia de Software Brasília, 2004.

Marshall, S., Mitchell, G. Applying SPICE to e-Learning: An e-Learning Maturity Model? In: $6^{\text {th }}$ Australasian Computing Education Conference (ACE2004), Dunedin. Vol.30. 2004. 
Russel, S.Norvig,P.Artificial Intelligence:A Modern Approach. New Jersey:Prentice Hall, 1995.

Salton, G. (1968). Automatic Information Organization and Retrieval. McGraw-Hill, New York.

Salton, G., Yang, C. E Wong, A. A vector-space model for automatic indexing. In: Communications of the ACM. 1975.

Shang, Yi. Shi, Hongchi. Chen, Su-Shing. "An intelligent distributed environment for active learning". In: ACM Journal of Educational Resources in Computing (JERIC). Vol. 1, No. 2, Summer 2001, Article 4, 17 pages. ISSN:1531-4278. New York: ACM Press. 2001.

Santos, T. L. T., Almeida, J. F. Obtenção dos Coeficientes de Transmissão e de Reflexão de Ondas Eletromagnéticas em Regiões Descontínuas Utilizando os Recursos Computacionais. In: III Congresso Nacional de Iniciação Científica CONIC, São Paulo, 2003.

Tavares, O. L., Brito, S. R., Souza, R. S., Menezes, C. S. Ambiente de apoio à mediação de aprendizagem: Uma abordagem orientada por processos e projetos. Revista de Informática na Educação. 2001.

Techniquest. Another look at science. http://www.tquest.org.uk. 2005. 